\title{
ON THE ZEROES OF PROFILES
}

\author{
W. D. WALLIS \\ (Received 23 May 1988; revised 29 July 1988)
}

Communicated by Louis Caccetta

\begin{abstract}
The $k$-profile of an Hadamard matrix of order $n$ is a function defined on the integers $0,1, \ldots, n$. If $k$ is even, $k$-profiles have been used in investigations of Hadamard equivalence. In this paper it is shown that the $k$-profile of an Hadamard matrix of order $n$ ( $k$ even) has non-zero terms only in every eighth position. If $k$ is divisible by 4 , the non-zero positions are those congruent to $n$ (modulo 8).
\end{abstract}

1980 Mathematics subject classification (Amer. Math. Soc.) (1985 Revision): 05 B 20.

\section{Introduction}

A general discussion of Hadamard matrices can be found in various places, such as [2], [4], [5]. We defined the profile, or 4-profile, in [1]. Given an Hadamard matrix $H=\left(h_{i j}\right)$, define

$$
P_{i j k l}=\left|\sum_{\alpha} h_{i \alpha} h_{j \alpha} h_{k \alpha} h_{l \alpha}\right| .
$$

and define $\pi(t)$ to equal the number of 4-sets $\{i, j, k, l\}$ such that $P_{i j k l}=t$. The function $\pi$ is the 4-profile of $H$. More generally one can define 6-profiles, 8 -profiles, and $k$-profiles for any larger even integer $k$. (The case of odd $k$, and the case $k=2$, are not interesting.) These profiles have been used in studying Hadamard equivalence (see [1], [3]).

(C) 1989 Australian Mathematical Society $0263-6115 / 89 \$ A 2.00+0.00$ 
It is easy to show (see [1]) that every $P_{i j k l}$ is congruent to $n$ modulo 8; so, for a 4-profile of an $n \times n$ Hadamard matrix, $\pi(t)=0$ unless $t \equiv n(\bmod$ 8). Empirical evidence suggests a similar result for $k$-profiles in general (only one in eight of the integers give a non-zero value of the function) but clearly the proof in [1] does not generalize to $k$ greater than 4; it depends on solving a set of equations, and the number of variables grows more quickly than the number of equations when $k$ increases. Moreover the behavior of the profile function is not easy to predict: the 6-profile of an Hadamard matrix of order 20 has its non-zero entries at $t=0,8$ and 16 (not $t \equiv n$ ), but the 6-profiles of Hadamard matrices of order 24 have zero entries for $t=0,8,16$ and 24 $(t \equiv n)$.

Our aim in this paper is to clarify this aspect of the behavior of profiles.

\section{Binomial coefficients}

We need some well-known preliminary results on binomial coefficients. Proofs are included for completeness; various alternative proofs are known.

LEMMA 1. For any positive integer $t$,

$$
\left(\begin{array}{c}
4 t \\
0
\end{array}\right)+\left(\begin{array}{c}
4 t \\
4
\end{array}\right)+\left(\begin{array}{c}
4 t \\
8
\end{array}\right)+\cdots+\left(\begin{array}{c}
4 t \\
4 t
\end{array}\right)
$$

is even.

Proof. Since $\left(\begin{array}{c}4 t \\ 0\end{array}\right)=\left(\begin{array}{c}4 t \\ 4 t\end{array}\right),\left(\begin{array}{c}4 t \\ 4\end{array}\right)=\left(\begin{array}{c}4 t \\ 4 t-4\end{array}\right)$, and so on, we can pair off the terms in the sum with equals. When $t$ is odd, this process exhausts the terms, so the sum is even. When $t$ is even, $\left(\begin{array}{c}4 t \\ 2 t\end{array}\right)$ is left unpaired; but

$$
\left(\begin{array}{l}
4 t \\
2 t
\end{array}\right)=\left(\begin{array}{l}
4 t-1 \\
2 t-1
\end{array}\right)+\left(\begin{array}{c}
4 t-1 \\
2 t
\end{array}\right)=\left(\begin{array}{l}
4 t-1 \\
2 t-1
\end{array}\right)+\left(\begin{array}{l}
4 t-1 \\
2 t-1
\end{array}\right)
$$

which is even.

LEMMA 2. If $t$ is any positive integer, then

$$
1+\left(\begin{array}{c}
4 t+2 \\
4
\end{array}\right)+\left(\begin{array}{c}
4 t+2 \\
8
\end{array}\right)+\cdots+\left(\begin{array}{c}
4 t+2 \\
4 t
\end{array}\right)
$$

is even.

Proof. Consider

$$
S=\left(\begin{array}{c}
4 t+2 \\
0
\end{array}\right)+\left(\begin{array}{c}
4 t+2 \\
2
\end{array}\right)+\left(\begin{array}{c}
4 t+2 \\
4
\end{array}\right)+\cdots+\left(\begin{array}{c}
4 t+2 \\
4 t+2
\end{array}\right)
$$


Using the rule

$$
\left(\begin{array}{c}
4 t+2 \\
i
\end{array}\right)=\left(\begin{array}{c}
4 t+1 \\
i
\end{array}\right)+\left(\begin{array}{c}
4 t+1 \\
i-1
\end{array}\right)
$$

we see that

$$
\begin{aligned}
S= & +\left[\left(\begin{array}{c}
4 t+1 \\
1
\end{array}\right)+\left(\begin{array}{c}
4 t+1 \\
2
\end{array}\right)\right]+\left[\left(\begin{array}{c}
4 t+1 \\
3
\end{array}\right)+\left(\begin{array}{c}
4 t+1 \\
4
\end{array}\right)\right] \\
& +\cdots+\left(\begin{array}{c}
4 t+1 \\
4 t+1
\end{array}\right)=2^{4 t+1} .
\end{aligned}
$$

This is divisible by 4 . Now

$$
\begin{aligned}
S= & \left(\begin{array}{c}
4 t+2 \\
0
\end{array}\right)+\left(\begin{array}{c}
4 t+2 \\
4
\end{array}\right)+\cdots+\left(\begin{array}{c}
4 t+2 \\
4 t
\end{array}\right) \\
& +\left(\begin{array}{c}
4 t+2 \\
4 t+2
\end{array}\right)+\left(\begin{array}{c}
4 t+2 \\
4 t-2
\end{array}\right)+\cdots+\left(\begin{array}{c}
4 t+2 \\
2
\end{array}\right)
\end{aligned}
$$

which equals twice the required sum.

\section{Symmetric functions}

Suppose the $m$ variables $x_{1}, x_{2}, \ldots, x_{m}$ each satisfy $x_{i}^{2}=1$. Write $X=$ $\left\{x_{1}, x_{2}, \ldots, x_{m}\right\}$, and define $\sigma_{j}$ to be the $j$ th symmetric function on $X$ :

$$
\sigma_{j}=\sum_{Y} \prod_{x \in Y} x
$$

where $Y$ ranges through the distinct $j=$ subsets of $X$. In particular $\sigma_{0}=1$. Then $\sigma_{j}$ is the sum of $n_{j}=\left(\begin{array}{c}m \\ j\end{array}\right)$ terms.

LeMma 3. $\sigma_{1} \sigma_{j}=(j+1) \sigma_{j+1}+(m-j+1) \sigma_{j-1}$.

Proof. Since $\sigma_{j}$ contains $\left(\begin{array}{c}m \\ j\end{array}\right)$ terms, so does $x_{i} \sigma_{j}$; of these $\left(\begin{array}{c}m-1 \\ j\end{array}\right)$ contain no repeated terms and $\left(\begin{array}{c}m-1 \\ j-1\end{array}\right)$ contain an $x_{i}^{2}$. So $x_{i} \sigma_{j}$ contains $\left(\begin{array}{c}m-1 \\ j\end{array}\right)$ terms of length $j+1$ and $\left(\begin{array}{c}m-1 \\ j-1\end{array}\right)$ terms of length $j-1$. Consequently $\sigma_{1} \sigma_{j}$ contains $m\left(\begin{array}{c}m-1 \\ j\end{array}\right)$ terms of length $j+1$ and $m\left(\begin{array}{c}m-1 \\ j-1\end{array}\right)$ terms of length $j-1$. By symmetry it is clear that $\sigma_{1} \sigma_{j}=A \sigma_{j+1}+B \sigma_{j-1}$ for some integers $A$ and $B$; therefore $A n_{j+1}=m\left(\begin{array}{c}m-1 \\ j\end{array}\right), B n_{j-1}=m\left(\begin{array}{c}m-1 \\ j-1\end{array}\right)$, and it follows that $A=j+1, B=$ $m-j+1$, giving the lemma.

COROLLARY. If $x_{0}$ is another variable satisfying $x_{0}^{2}=1$, then

$$
\begin{gathered}
\left(x_{0}+x_{1}\right)\left(x_{0}+x_{2}\right) \cdots\left(x_{0}+x_{m}\right)\left(x_{1}+x_{2}+\cdots+x_{m}\right) \\
=m\left(\sigma_{0}+x_{0} \sigma_{1}+\sigma_{2}+x_{0} \sigma_{3}+\cdots+x_{0}^{m} \sigma_{m}\right) .
\end{gathered}
$$


Proof. The left-hand side equals $\left(x_{0}^{m}+x_{0}^{m-1} \sigma_{1}+x_{0}^{m-2} \sigma_{2}+\cdots+\sigma_{m}\right) \sigma_{1}$. The result now follows from repeated application of Lemma 3.

\section{Profiles}

THEOREM. Suppose $H$ is an Hadamard matrix of side $n, n \geq 4$, and suppose $k$ is even. Then the generalized inner product of $k$ rows,

$$
P_{i_{1} i_{2} \cdots i_{k}}=\sum_{j=1}^{n} h_{i_{1} j} h_{i_{2} j} \cdots h_{i_{k} j}
$$

is congruent to $n$ modulo 8 when 4 divides $k$, and is congruent to 0 modulo 8 when $k$ is congruent to 2 modulo 4 .

Proof. We write $X_{j}=\left\{h_{i_{2} j}, h_{i_{3} j}, \ldots h_{i_{k} j}\right\}$, and denote the $i$ th symmetric function on $X_{j}$ by $\sigma_{i j}$.

Assume $k \geq 4$; consider

$$
Q_{j k}=\left(h_{i_{1} j}+h_{i_{2} j}\right)\left(h_{i_{1} j}+h_{i_{3} j}\right) \cdots\left(h_{i_{1} j}+h_{i_{k} j}\right)\left(h_{i_{2} j}+h_{i_{3} j}+\cdots+h_{i_{k} j}\right) .
$$

As $k \geq 4, Q_{j k}$ is divisible by 8 . On the other hand, by the Corollary to Lemma 3,

$$
Q_{j k}=(k-1)\left(\sigma_{0 j}+h_{i_{1} j} \sigma_{1 j}+\sigma_{2 j}+h_{i_{1} j} \sigma_{3 j}+\cdots+h_{i_{1} j} \sigma_{k-1, j}\right)
$$

Now

$$
\sum_{j=1}^{n} Q_{j k}=(k-1)\left(n+\sum h_{i_{1} j} \sigma_{i j}+\sum \sigma_{2 j}+\cdots+\sum h_{i_{1} j} \sigma_{k-1, j}\right) .
$$

We now reduce modulo 8 . Since each $Q_{j k}$ is zero $(\bmod 8)$, the sum is zero. Since $n$ is the order of an Hadamard matrix and $n \geq 4, n$ is divisible by 4 , so $n \equiv-n(\bmod 8)$. Finally $k-1$ is odd. So

$$
\sum h_{i_{1} j} \sigma_{1 j}+\sum \sigma_{2 j}+\cdots+\sum h_{i_{1} j} \sigma_{k-1, j} \equiv n(\bmod 8) .
$$

We now write $f(k)$ for the residue class of $P_{i_{1} i_{2} \cdots i_{k}}(\bmod 8)$, and define $f(0) \equiv n$. We prove by induction that $f(k)$ is well defined (in other words, the residue class depends only on $k$ ), and that

$$
\sum_{2 \mid \alpha}\left(\begin{array}{l}
k \\
\alpha
\end{array}\right) f(\alpha) \equiv 0 \quad(\bmod 8) .
$$


When $k=0$ the result is obvious. In general, if $2 t<k, \sum h_{i, j} \sigma_{2 t-1, j}+\sum \sigma_{2 t, j}$ is the sum of $n_{2 t-1}+n_{2 t}$ terms, each of which equals an inner product of $2 t$ rows, so by the hypothesis that $f(2 t)$ is well defined the sum is congruent to $\left(n_{2 t-1}+n_{2 t}\right) f(2 t)$; and

$$
n_{2 t-1}+n_{2 t}=\left(\begin{array}{c}
k-1 \\
2 t-1
\end{array}\right)+\left(\begin{array}{c}
k-1 \\
2 t
\end{array}\right)=\left(\begin{array}{c}
k \\
2 t
\end{array}\right) .
$$

Moreover $P_{i_{1} i_{2} \cdots i_{k}}=\sum h_{i, j} \sigma_{k-1, j}$. So (2) yields

$$
\left(\begin{array}{l}
k \\
2
\end{array}\right) f(2)+\left(\begin{array}{l}
k \\
4
\end{array}\right) f(4)+\cdots+f(k) \equiv n \quad(\bmod 8),
$$

which becomes (1) when we observe that $\left(\begin{array}{l}k \\ 0\end{array}\right) f(0) \equiv n \equiv-n(\bmod 8)$.

We now show, by induction again, that $f(k) \equiv n$ when 4 divides $k$ and is congruent to 0 otherwise. Suppose this is true of $f(\alpha)$ for all values of $\alpha$ less than $k$. Then

$$
-f(k) \equiv \sum\left(\begin{array}{l}
k \\
\alpha
\end{array}\right) f(\alpha) \equiv\left[\sum_{1}\left(\begin{array}{l}
k \\
\alpha
\end{array}\right)\right] \cdot n+\left[\sum_{2}\left(\begin{array}{l}
k \\
\alpha
\end{array}\right)\right] \cdot 0
$$

where $\sum_{1}$ is over $\alpha$ divisible by 4 and $\sum_{2}$ over $\alpha$ congruent to $2(\bmod 4), 0 \leq$ $\alpha<k$.

If 4 divides $k$ then by Lemma $1, \sum_{1}\left(\begin{array}{l}4 \\ \alpha\end{array}\right)+\left(\begin{array}{l}k \\ k\end{array}\right)$ is even, so $\sum_{1}\left(\begin{array}{l}k \\ \alpha\end{array}\right)$ is odd and the expression is congruent to $n$ modulo 8 . If 4 does not divide $k$ then $\sum_{1}\left(\begin{array}{l}k \\ \alpha\end{array}\right)$ is even by Lemma 2 and the expression is congruent to zero. The other bracketed term is zero. Since $-n \equiv n(\bmod 8)$, the negative sign is irrelevant.

COROLLARY. If $k \equiv 0(\bmod 4)$ then the $k$-profile of an Hadamard matrix has non-zero terms only in positions congruent to $n(\bmod 8)$. If $k \equiv 2$ (mod 4) then the $k$-profile of an Hadamard matrix has non-zero terms only in positions congruent to $0(\bmod 8)$.

\section{References}

[1] J. Cooper, J. Milas and W. D. Wallis, 'Hadamard equivalence,' Combinatorial Mathematics, 126-135 (Springer-Verlag, Heidelberg, 1978).

[2] M. Hall, Jr., Combinatorial theory (2nd ed., John Wiley, New York, 1986). 
[3] C. Lin and W. D. Wallis, Profiles of Hadamard matrices of order 24, Congr. Numer. 66 (1988), 93-102.

[4] W. D. Wallis, Combinatorial designs (Marcel Dekker, New York, 1988).

[5] W. D. Wallis, A. P. Street and J. S. Wallis, Combinatorics: Room squares, sum-free sets, Hadamard matrices (Springer-Verlag, Heidelberg, 1972).

\author{
Department of Mathematics \\ Southern Illinois University \\ Carbondale, Illinois 62901-4408 \\ U.S.A
}

